\title{
A continued role for signalling functions in the early evolution of feathers
}

\author{
Graeme D Ruxton ${ }^{1}$, Walter S Persons ${ }^{2}$, Philip J Currie ${ }^{2}$
}

\section{School of Biology, University of St Andrews, St Andrews KY16 9TH, UK. gr41@st-andrews.ac.uk}

\section{Department of Biological Sciences, University of Alberta, Edmonton, Canada}

Running head: signalling in the early evolution of feathers

Keywords: flight, thermoregulation, bristles, down, plumage, feathered dinosaurs, evolution of birds

\begin{abstract}
Persons and Currie (2015) argued against either flight, thermoregulation, or signalling as a functional benefit driving the earliest evolution of feathers; rather, they favoured simple feathers having an initial tactile sensory function, which changed to a thermoregulatory function as density increased. Here, we explore the relative merits of early simple feathers that may have originated as tactile sensors progressing instead towards a signalling, rather than (or in addition to), a thermoregulatory function. We suggest that signalling could act in concert with a sensory function more naturally than could thermoregulation. As such, the dismissal of a possible signalling function and the presumption that an initial sensory function led directly to a thermoregulatory function (implicit in the title "bristles before down") are premature.
\end{abstract}

This article has been accepted for publication and undergone full peer review but has not been through the copyediting, typesetting, pagination and proofreading process, which may lead to differences between this version and the Version of Record. Please cite this article as doi: 10.1111/evo.13178.

This article is protected by copyright. All rights reserved. 


\section{Main text}

The three most obvious functions of feathers in modern birds are flight, thermoregulation and signalling. Persons and Currie (2015) argued that none of these mechanisms offer a satisfying functional explanation for the earliest origin of feathers. It is relatively uncontroversial to state that hair-like proto-feathers did not, and could not, provide an aerodynamic function given that modern flight feathers require a number of complex features to form effective gliding surfaces or aerofoils, and given that feathers are widespread in phylogenetically more basal and certainly-flightless dinosaurian lineages (some only very distantly related to birds). Persons \& Currie (2015) argued that thermal insulation is unlikely to offer a full functional explanation either, since "like mammalian hair, simple feathers could serve as insulation only when present in sufficiently high concentrations". They also did not favour display as a likely initial driver of the earliest proto-feathers: "sexual display is an explanation that can be theorised to have played a role in the evolution of nearly any outwardly visible feature. As such, sexual display constitutes something of a default explanation ..... there is nothing particular about the form of simple feathers that supports the sexual display theory."

Having dismissed the three most-considered mechanisms, Persons \& Currie (2015) argued that feathers may have originated to serve as tactile sensors. Facial bristles are prevalent in modern birds, and have a simple form featuring a single relatively-stiff rachis with little or no venation at their distal tip. As such, the basic forms (though not the developmental homology) of these bristles are more similar to the structure of proto-feathers than modern flight or down feathers are. In modern birds, bristles primarily serve a tactile sensory 
function. Such a function does not require a dense covering of bristles, unlike thermoregulation or flight. The key to the evolutionary scenario that they envisage is that "it is not difficult to imagine how the first simple feather, or a sparse arrangement of single feathers appeared on the face of a dinosaur ... and provided immediate slight selective benefit in the form of increased tactile sensitivity." The argument of Persons and Currie (2015) remains theoretical and awaits direct fossil evidence of definitive tactile bristles in a primitive and otherwise feather-less dinosaur.

Nonetheless, all the authors of this consensus think that a strong case has been made for the possibility that facial bristles evolved in archosaurs through a tactile sensory function.

However, the first author has raised several points regarding whether functions other than thermoregulation (most obviously display and more generally signalling) might also have played a significant role in the spread of feathers from singular filaments on the face, to a coat across the entirety of the body. While we three authors are not unanimous in thinking signalling offers a better functional route for the spread of feathers in non-avian dinosaurs, we are in agreement that both theories have distinct merits and that the signalling route is worth greater consideration than that previously afforded it by Persons and Currie (2015). First, consider how Persons and Currie (2015) explained the spread of feathers across the body: "Evolutionary pressures could then gradually favour more and longer bristles for the same [sensory] function. Concentrations of bristles ... would begin to offer benefits as dust shields, and once a high enough concentration of bristles was reached they would begin to provide a small degree of insulation. As facial feathers, bristles would have been optimally positioned to insulate ... an animal's head. In the final step, a high concentration of simple facial feathers with the primary function of thermal insulation would develop and eventually spread to encompass the rest of the body." While it is true that bristles around the eyes and 
nostrils do appear to function in part as dust shields, in other respects there are important caveats to this argument.

The pathway described above explicitly suggests a transition in primary selective pressure whereby, on at least part of the head, a covering of bristles evolved for tactile benefits, but then became so dense that the primary selective benefit changed to thermoregulation. In modern birds, bristles considered to have a primarily sensory function are generally confined to the front of the head and it is difficult to see how bristles elsewhere on the head would be advantageous from a sensory perspective. Bristles certainly occur in one or more tufts and it is possible to envision multiple concentrated bristles affording the benefit of more accurate localisation of tactile stimuli and greater perception of stimuli movement relative to the head. However, it is also likely that there would be diminishing returns in terms of the benefits from ever-greater densities of bristles.

Moreover, in certain regards, selection for sensory and thermal benefits may have been intrinsically antagonistic. To best serve as transmitters of tactile information, bristles must be relatively stiff, whereas coverings whose primary function is thermoregulation are generally soft and downy in order to trap small pockets of air. Similarly, although bristles frequently grow in clusters, they tend to radiate away from one another and function best when their tips are not at risk of touching and interfering with each other. Thus, the function of bristles is intrinsically at odds with high bristle density. As such, it is questionable whether sensory benefits alone could drive the evolution of a density of bristles sufficient to then offer thermoregulation benefits and, in turn, to drive further increases in feather density.

Next, there is the hypothesis that "a high concentration of simple facial feathers with the primary function of thermal insulation would develop and eventually spread to encompass the rest of the body." Imagine that the animal in question has evolved to a stage where the head is 
covered in simple feathers whose primary functional benefit is now thermal insulation. How might feathers then spread across the body? It is possible to imagine the presence of feathers spreading to the anterior of the neck immediately adjacent to the area of the head where feathers currently grow. If this enhanced covering is beneficial, then the adaptation for a more extensive feather covering will be selected. A thick covering could progressively spread out from the head in this way and, eventually, progressive expansion would lead to feathers covering the whole body. However, this model makes the prediction that progressive expansion would occur extending out in a continuous wave across the body starting from the head region. There is no empirical support, either from the fossil record or developmental studies, for this pattern of progressive spread.

An additional or alternative signalling function circumvents many of the limitations of the "tactile then thermoregulatory" model described above. Firstly, there is no obvious trade-off between signalling and thermoregulation, as there is between tactile sensory functioning and thermoregulation. This should facilitate any downstream selection for thermoregulation, which could be achieved without necessarily incurring a cost in reduced signalling effectiveness. Secondly, proto-feathers could augment a display no matter how thin and/or constrained their distribution, and that signalling function could strengthen as either the density and/or coverage of the proto-feathers increased. Finally, signalling function does not require a continuous coverage of proto-feathers, and there is no reason why proto-feathers could not evolve on separate parts of the body with naked parts interposed.

Just as importantly, there is no need to think of signalling and tactile functions as mutually exclusive. Indeed, stiffened and prominently-projecting integumentary structures are entirely consistent with potential signalling functions. If proto-feathers evolved initially on the face for tactile reasons, nothing prohibits those structures from also serving in signalling, 
particularly since the face is often the focus of visual attention in social interactions. Having developed this dual role, it is relatively easy to envisage the spread of such structures to other body parts driven by enhanced signalling benefits but with possible ancillary tactile sensory benefits (or vice versa). The constraints on such spread are much less for a primarily signalling function than a primarily thermoregulatory one. Specifically, increased feather coverage could be selected through a signalling function even if the new area of coverage was discontinuous from previous areas of coverage, and even if the initial coverage in the new area was sparse - both in stark contrast to the requirements of thermoregulation.

Finally, it is worth noting that a dense covering of long and relatively-stiff integumentary fibres (which may or may not be homologous with the feathers of the avian line) are known from the tail of the ornithischian dinosaur Psittacosaurus (Mayr et al. 2002). These tail fibres appear oversized for a role in thermoregulation and are best explained as signalling structures. Skin fossils confirm that the rest of a Psittacosaurus body was devoid of an insulating integumentary coat, and thus Psittacosaurus offers one example of stiffened fibrous signalling integuments evolving in a dinosaur with no evidence of accompanying or pre-existing thermoregulatory function.

\section{Acknowledgment}

We thank Prof. Mohamed Noor for encouraging us to work to find a consensus position, and both he and Dr. Joseph Tobias for valuable comments that improved our submission.

\section{References}


Mayr, G., Peters, S. D., Plodowski, G. and Vogel, O., 2002. Bristle-like integumentary structures at the tail of the horned dinosaur Psittacosaurus. Naturwissenschaften, 89(8): 361365.

Persons, W. S. and Currie, P. J., 2015. Bristles before down: A new perspective on the functional origin of feathers. Evolution 69(4): 857-862. 